\title{
Electronic application to evaluate the driver's activity on the polluting emissions of road traffic
}

\author{
D. Pérez, F. Espinosa, M. Mazo, J. A. Jiménez, E. Santiso, \\ A. Gardel \& A. M. Wefky \\ Department of Electronics, University of Alcalá, Spain
}

\begin{abstract}
The polluting emissions (gases and particles) produced by the traffic of automobiles are directly related to the activity of vehicle, but they are also affected by the route conditions and moreover, by the driver's behavior. However, PAMS commercial systems do not usually include elements to register this last component. This article presents an electronic system, specially designed to evaluate, ad-hoc, the effect of driver's activity on polluting emissions. This electronic application integrates a hardware and a software component, both designed concerning MIVECO research project. From the hardware component the sensorial part stands out, formed by potentiometers connected to the pedals that control the vehicle and the inertial device, which allows one to evaluate the instantaneous accelerations in the $x-y-z-a x e s$ as well as the turns with respect to these axes. Once the signals are conditioned and acquired, the software component processes them for on-line monitoring in a GUI and stores them in a database to facilitate its evaluation off-line. This electronic application has two important properties: it can be incorporated in any vehicle of the market (light or heavy, diesel or gasoline, pre or post-eobd) and it allows the capture and registration of information about the driver's activity synchronously with PEMS (gases and/or particles) systems. The work includes experimental results obtained in an urban circuit in the city of Madrid.
\end{abstract}

Keywords: driver's behavior, PAMS, eco-driving, multi-sensorial system, onboard electronic system. 


\section{Introduction}

According to various studies realized by distinct investigation groups [1-5] and official organizations from different countries [6-12], it was concluded that both the surrounding environment and the driving form affect both the fuel consumption and the gaseous as well as particles pollutant emissions of the vehicle.

Other studies show that relationship using only the speed measurement to determine if the driving is more efficient in terms of the emission of the pollutant gases $\left(\mathrm{CO}, \mathrm{HC}, \mathrm{NO}_{\mathrm{x}}, \mathrm{CO}_{2}\right)[13,14]$.

In addition to savings in pollutant emissions, ecological driving produces a multitude of enhancements such as an average saving of $15 \%$ of fuel, less noise pollution, reduction in the risk of accidents of between 10 and $25 \%$, reduction in maintenance costs of the vehicle: brakes, clutch, gearbox and engine, and increased comfort for the driver and the passengers [14].

In this context the challenge is to have onboard equipment that records information from the driver's activity in synchronisation with complementary systems for measuring emission (PEMS).

A solution that integrates two types of measuring, direct and indirect, the activity of the driver is proposed in this paper. On one hand, the direct action on the pedals to control the vehicle is measured. On the other hand, motion variables, as linear acceleration, speed and inclination of the vehicle in 3 axes, are recorded using an inertial sensor.

\section{Methodology}

An in-vehicle electronic system was designed to measure driver activity. The designed system consists of three basic subsystems, as shown in Fig. 1.

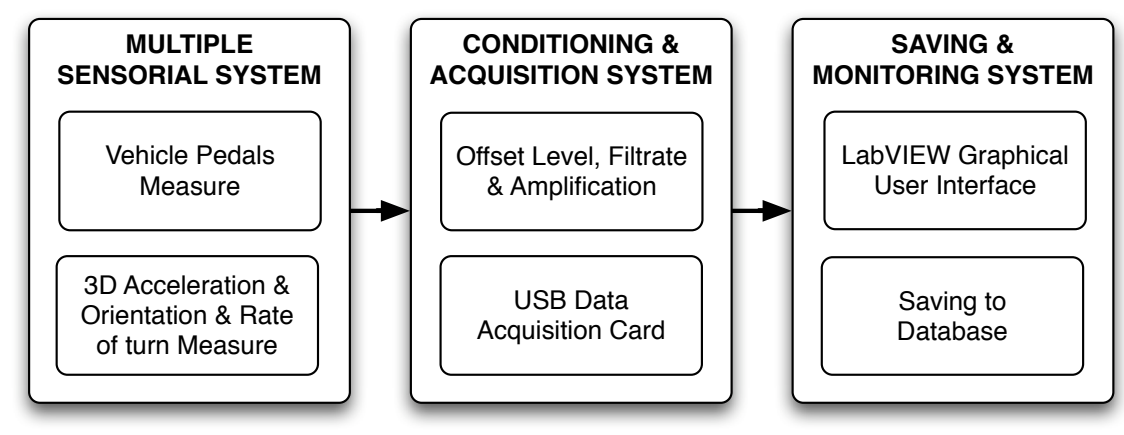

Figure 1: Block diagram of the global system.

The first subsystem contains the study of selection and placement of the sensory system and its measurement characteristics. The second subsystem contains the development and acquisition of signals from the sensors. And the 
third subsystem is responsible for monitoring and recording information from sensors in a database on a laptop computer.

\subsection{Sensorial system}

The sensorial system consists of two parts that enable the measurement of the driver behavior. On one hand, three potentiometers are used to provide a signal proportional to the action of the driver on the three pedals of the vehicle. On the other hand, an inertial measurement unit is used, to provide signals proportional to acceleration, direction, and speed of the vehicle on the XYZ-axes.

\subsubsection{Potentiometers measurement characteristics}

Three ASM WS42C displacement potentiometers were used to measure the activity of the pedals [15]. Such potentiometers are based on the variation of the resistance in proportion with the displacement. That displacement is produced by stretching the wire rolled around a pulley and controlled by a spring. When the force on the cable is released, it returns to its original position automatically.

The three potentiometers are fixed by a metal plate with screws to the internal part of the vehicle under the dashboard, specifically to the left of the pedals as shown in the left side of Fig. 2. In order not to bother the driver, each end of the three potentiometers is tied to a nylon thread which is attached to the upper part of each pedal at its other end as shown in the right side of Fig. 2.
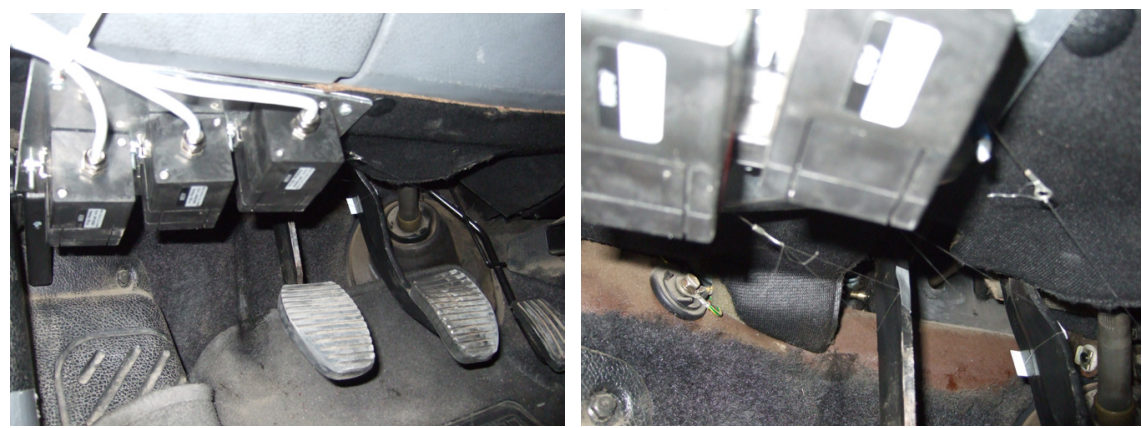

Figure 2: Potentiometers measurement system.

Once the system is set up, it is necessary to make an initial calibration to establish the dynamic range of the pedal displacement. That dynamic range depends on each vehicle model. In order to standardize and draw valid conclusions using different vehicles, the displacement values were normalized between 0 and $100 \%$ of the pedal path. Eqn. (1) uses the voltage resulting from each potentiometer which is proportional to the displacement to accomplish the normalization process.

$$
V_{\text {pedals }}(\%)=\frac{V_{\text {measured }}-V_{\min }}{V_{\max }-V_{\min }} \cdot 100
$$




\subsubsection{Inertial sensor measurement characteristics}

An inertial measurement sensor MTi of the company Xsens was used to indirectly measure the driver behaviour [16].

This device integrates gyroscope and accelerometers in the three axes and internally makes digital signal processing through a sensorial fusion process. It is able to provide the inclination (tilt), the angular speed and linear acceleration in the three axes. One of the advantages of such type of sensors is that due to its small and compact size, it can be easily installed in the vehicle. It is necessary to take into account making an initial reset of the system to compensate for the possible initial angle between the surface where the sensor is placed and the reference system of the vehicle. In order not to be affected by any possible unwanted vibrations, this sensor has been placed at the bottom of the vehicle as shown in the right side of Fig. 3.
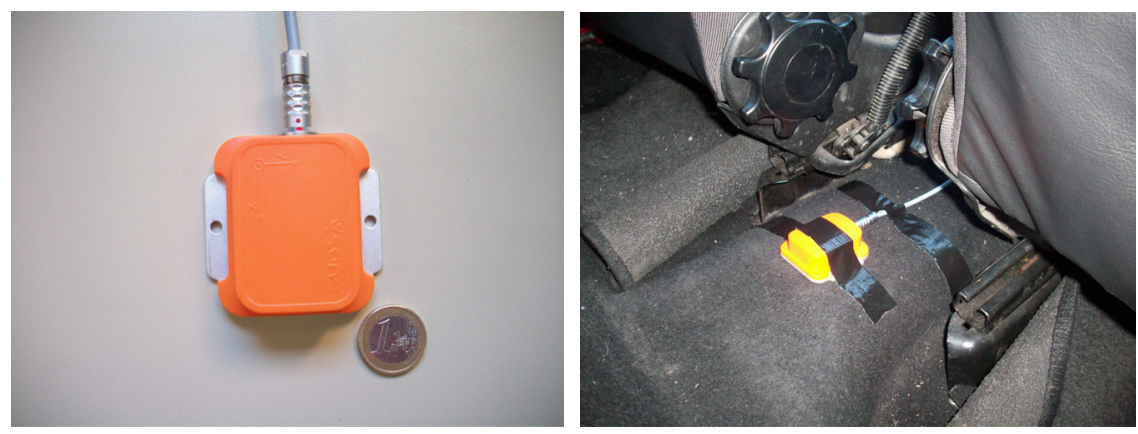

Figure 3: Inertial sensor measurement system.

\subsection{Conditioning and acquisition system}

The two sensory sources have different electrical properties. Consequently, two stages of data acquisition and conditioning were designed as shown below.

\subsubsection{Conditioning and acquisition characteristics of potentiometers}

It is necessary to make some signal conditioning operations on the output voltage of the potentiometers such that it occupies the entire dynamic range of the data acquisition card. Therefore, the signal conditioning module DAQP-BRIDGE-B, shown in Fig. 4, was used [17]. This module allows a gain adjustment by a differential amplifier. Moreover, it adjusts the offset of the signal. Finally, it contains a low pass filter to eliminate the possible high-frequency noise.

Once the signal was conditioned, a Wheatstone bridge configuration was used to measure small increments of voltage before proceeding to one of the channels of the data acquisition card.

The portable and high-speed data acquisition card USB NI-6211 of the company National Instruments was used to make the acquisition of the previously conditioned signals from the potentiometers. This card performs data communication with a laptop computer via a USB port and is fed through the 


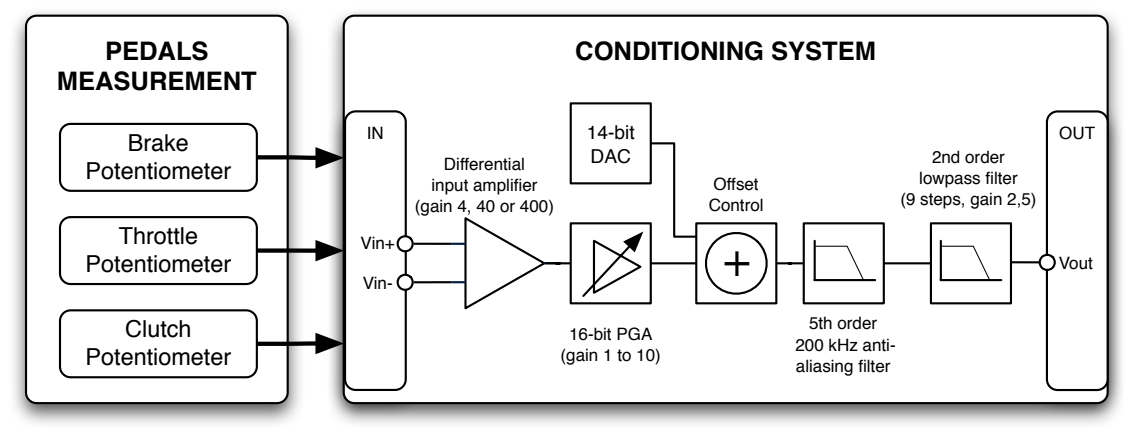

Figure 4: Potentiometers conditioning system.

same bus. A sampling period of $20 \mathrm{~ms}$, which is sufficient to represent the dynamics of the driver's action, was chosen to capture any quick action of the driver on any of the pedals.

\subsubsection{Inertial sensor conditioning and acquisition characteristics}

The inertial sensor is connected directly to the laptop that is responsible for storing the data acquired during a tour through another USB ports.

This sensor provides a built-in system of conditioning and acquisition. Therefore, it is not necessary to use one channel of the data acquisition card as in the case of potentiometers. This system, shown in Fig. 5, is software configurable and provides an output synchronized by a trigger. This trigger activates the acquisition and delivery of the desired data after a process of sensorial fusion. The sampling period of the system was also set to $20 \mathrm{~ms}$ to compare with the measurement system of the potentiometers.

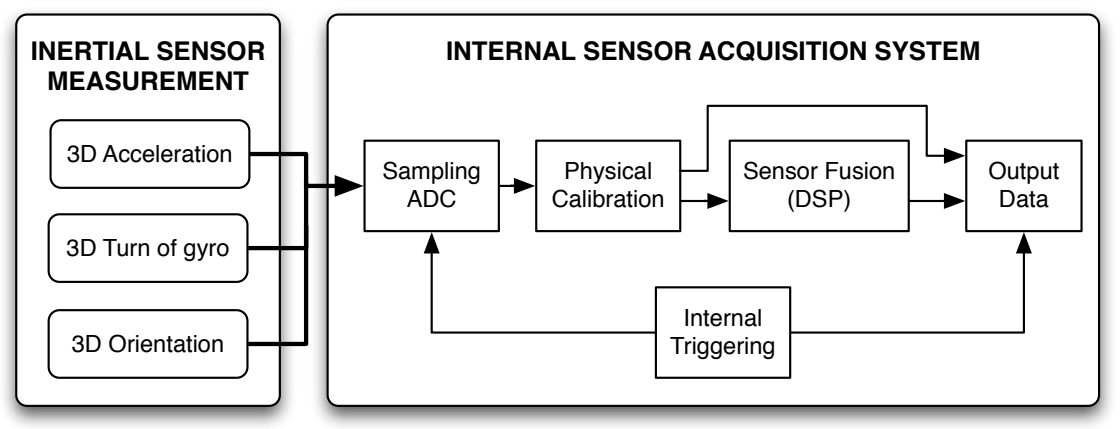

Figure 5: Inertial sensor acquisition system.

\subsection{Saving and monitoring system}

This system allows visualize and record the activity level of the driver. On one hand, the system shows the behavior of the variables measured by the sensorial 
system in real time. It was programmed with LabVIEW, an application that has a GUI as shown in Fig. 6 [19]. This application allows monitoring in a laptop onboard the vehicle.

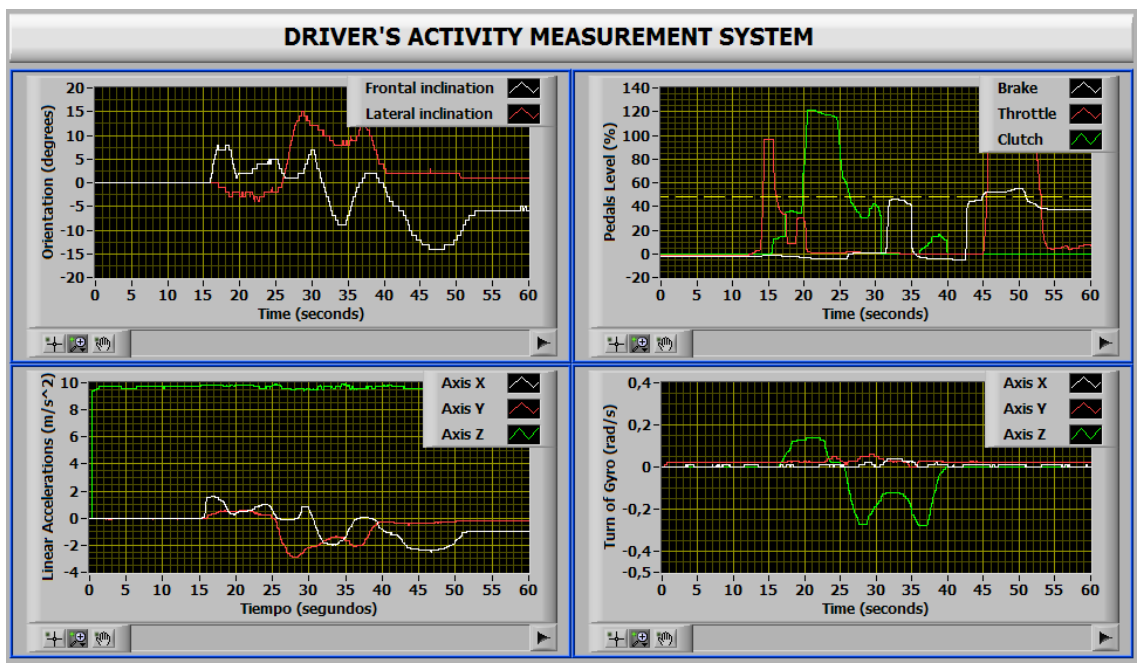

Figure 6: GUI for monitoring of the driver's activity.

Besides visualization, the designed software application allows recording of all signals captured by sensors in a database for off-line visualization and analysis. The database was designed using MySQL [20].

\section{Results and discussion}

The system has been designed to characterize the behavior of the driver by two complementary techniques whose results are detailed below.

\subsection{Driver's activity measurement with potentiometers}

This method allows determine the degree of drivers' activity on each of the three pedals in the car, i.e., accelerator, brake, and clutch, relative to the full scale of the pedal path.

The measurement of the accelerator and brake pedals helps decide whether the driver has a more or less aggressive driving behavior. In other words, he may drive with sudden and repeated accelerations and brakes. Or on the contrary, he may drive with soft and maintained accelerations and brakes.

The measurements of the three pedals for the driver realized during a tour in the center of Madrid are shown in Fig. 7. There are constant accelerations and brakes as well as gear changes are reflected in the variation of the clutch pedal.

Due to the complexity of analyzing the signals in urban environments where there are continuous changes in the actions of the pedals, it has employed. 


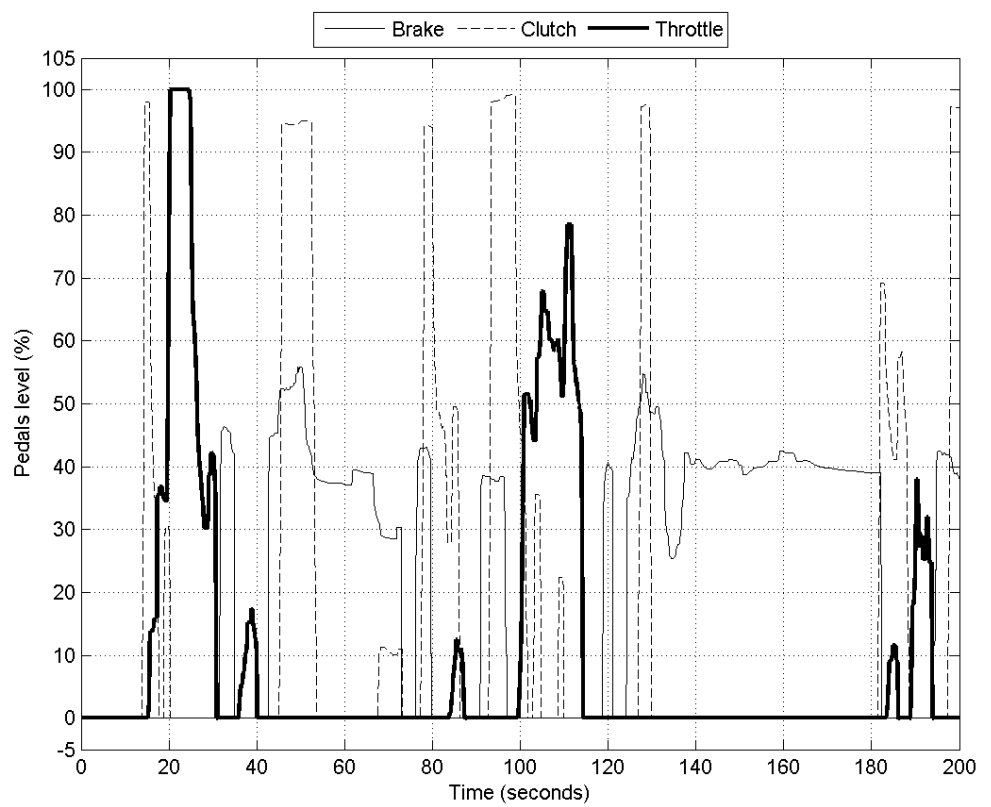

Figure 7: Vehicle pedals measurement in urban route.

A simple statistical analysis to evaluate the driver's behavior was made. A tour of the center of Madrid with an aggressive driving and a more relaxed one were conducted. As an example, the result obtained for the signal of the accelerator pedal position in the case of an aggressive driving with continuous and sudden accelerations as compared to a relaxed driving can be seen in the upper part of Fig. 8 .

A statistical analysis was performed to compare the two signals of the accelerator pedals of Fig. 8. Therefore, the mean and standard deviation of the signal were calculated, which are plotted in the lower part of Fig. 8, and then analytically in Table 1.

A higher value of the mean indicates that the values of the accelerator pedal while driving are of higher amplitude. On the other hand, a higher value of the standard deviation indicates the dispersion around the mean is higher, and consequently, there have been more pronounced changes in the accelerator pedal for the case study of aggressive driving.

On the other hand, the measurement of the clutch pedal should be analyzed in a different way from the brake and accelerator pedals. This signal helps determining whether the driver is travelling over low gear levels during a long time period or on the contrary, he uses high gear levels [14]. This criterion is also decisive to save fuel and extend engine life. 

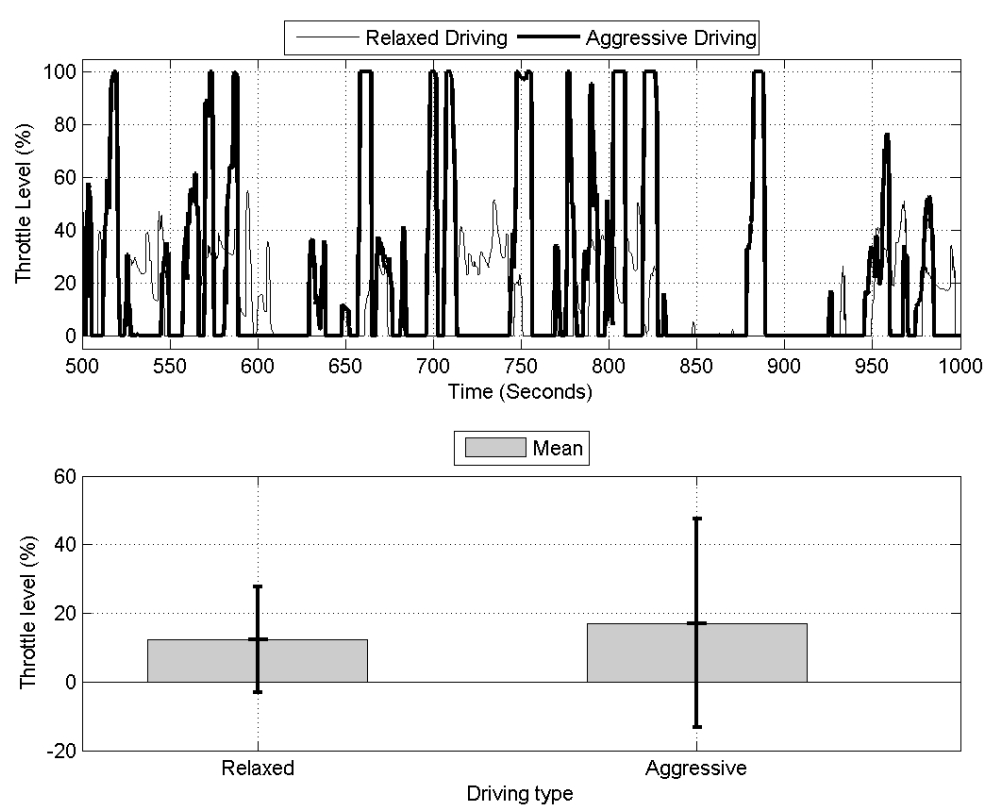

Figure 8: Accelerator level study in urban route.

Table 1: $\quad$ Throttle dispersion measure.

\begin{tabular}{|c|c|c|}
\hline Driving & Mean & Standard deviation \\
\hline Relaxed & 12.2768 & 15.4476 \\
\hline Aggressive & 17.0336 & 30.3452 \\
\hline
\end{tabular}

\subsection{Driver's activity measure with inertial sensor}

This method tells us in an indirect way when the vehicle slows down and speeds up paying attention to the measurement of acceleration in the $\mathrm{X}$-axis. This axis was chosen to represent the longitudinal movement of the vehicle. Increments in acceleration caused by sharp turns in curves, roundabouts or changing direction can be observed paying attention to the transverse axis and the $\mathrm{Y}$-axis.

The measurements of the pedals during the route stated previously for measuring the driver's activity by means of the pedals are shown in Fig. 9. Constant accelerations and decelerations in the $\mathrm{X}$ - and $\mathrm{Y}$-axes can also be observed here. It seems logical that the acceleration in the $\mathrm{Z}$-axis remains approximately constant around the value of gravity.

The measurement of the linear accelerations in the $\mathrm{X}$ - and $\mathrm{Y}$-axes principally allows deciding whether the driver has a more or less aggressive driving behavior, i.e., whether he drives with sharp accelerations in the forward direction of the vehicle, or he passes roundabouts in a more or less smooth manner. 


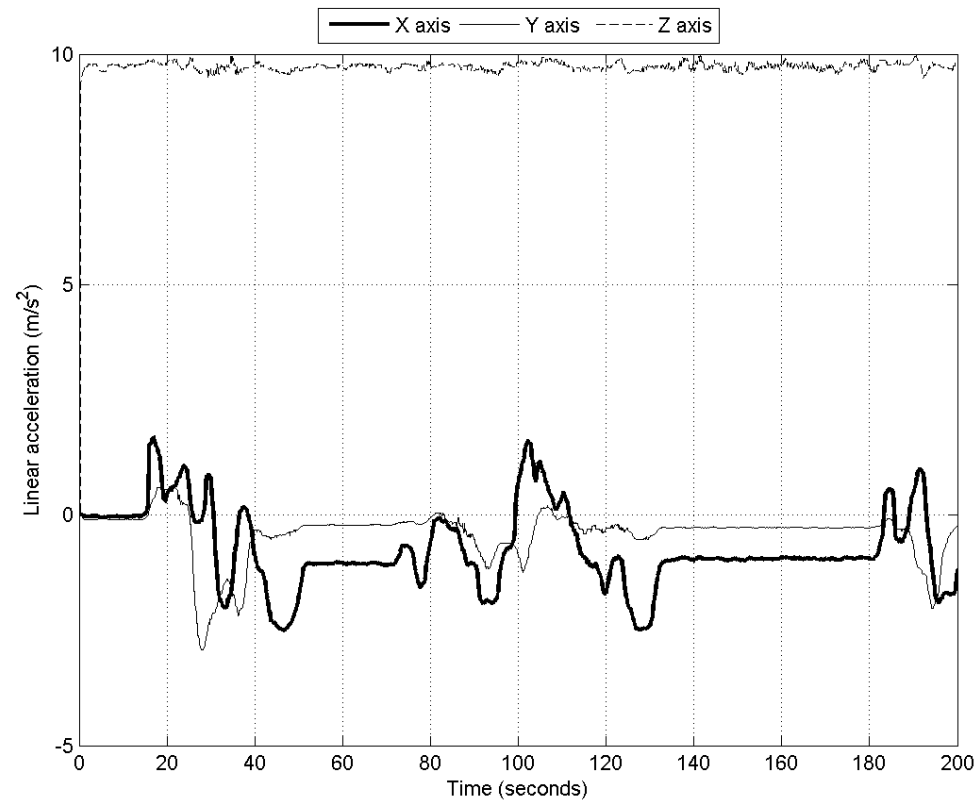

Figure 9: $\quad \mathrm{X}$-axis linear acceleration measurement in urban route.
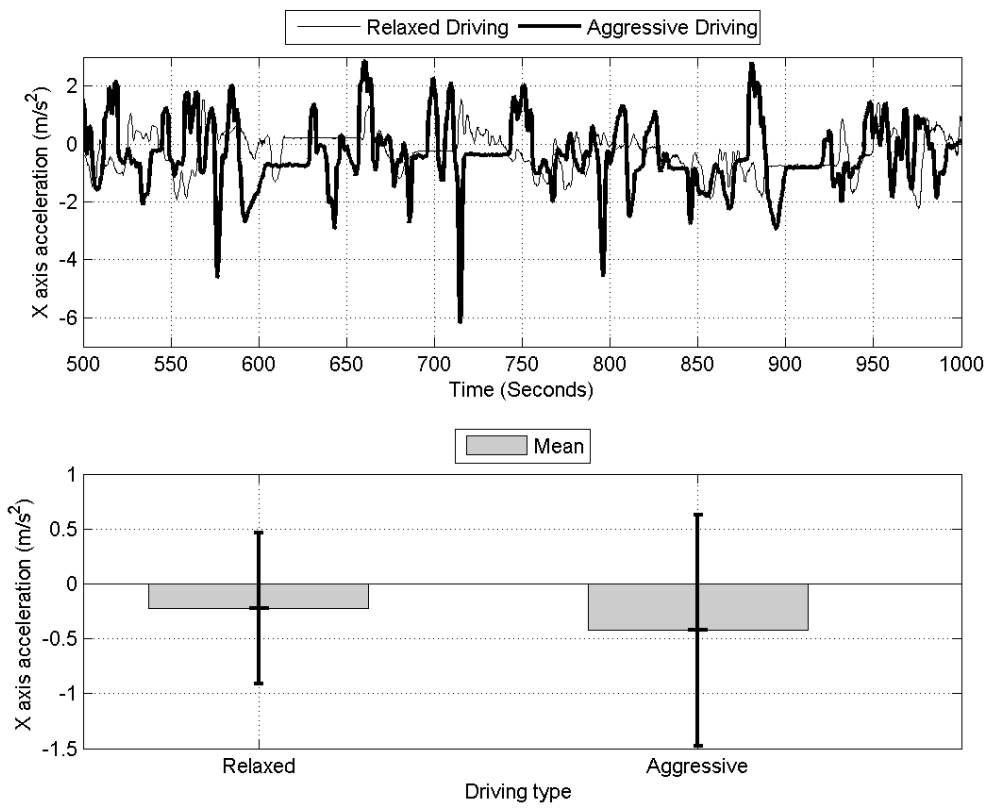

Figure 10: Axis $\mathrm{X}$ acceleration study in urban route. 
A simple statistical analysis to evaluate the driver's behavior was used due to the complexity of analyzing the signals in urban environments where there are continuous changes in the frontal and lateral accelerations of the vehicle. A tour of the center of Madrid with an aggressive driving and a more relaxed one was realized. For example, the result for the signal from the vehicle's linear acceleration in the $\mathrm{X}$-axis in case of aggressive driving as compared to a more relaxed driving can be seen in the upper part of Fig. 10.

A statistical analysis was performed to compare between the two signals of acceleration in the $\mathrm{X}$-axis in Fig. 10. We calculated the mean and standard deviation for both signals. The results are plotted in the lower part of Fig. 10 and illustrated numerically in Table 2 .

Table 2: $\quad$ Axis $\mathrm{X}$ acceleration dispersion.

\begin{tabular}{|c|c|c|}
\hline Driving & Mean & Standard deviation \\
\hline Relaxed & -0.2237 & 0.6877 \\
\hline Aggressive & -0.4251 & 1.0504 \\
\hline
\end{tabular}

In this case, the ideal value of the mean for the measurement of acceleration in the X-axis should be zero. The measured values are close to the ideal value. The standard deviation over the mean value in the ideal case of an aggressive driving is more than that of a relaxed driving. Because in the relaxed driving, there were minor amplitude changes in the $\mathrm{X}$-axis acceleration signal.

\section{Conclusions}

The activity of the driver is a key parameter in efficient driving in terms of fuel economy and pollution levels. Therefore, it is very useful to have an electronic system that records the variables associated with driving behavior synchronously with the measurement of gases and / or particles.

The designed and implemented system allows measuring the activity of the driver directly (using accelerator, clutch, and brake pedals activity) and indirectly (using instantaneous changes in the linear and angular movement of the vehicle). Simultaneously, the proposed system provides information on variables associated with more or less aggressive behavior of the driver.

The resulting electronic system in both its hardware and software components is autonomous, universal and easily integrated with other measurement applications in the car.

\section{References}

[1] Wada, T., Doi, S., Imai, K., Tsuru, N., Isasi, K., Kaneko, H., “Analysis of Drivers' Behaviors in Car Following Based on A Performance Index for Approach and Alienation", SAE Technical Papers, Document Number: 2007-01-0440, 2007. 
[2] Gordon, D., "Steering A New Course: Transportation, Energy, and the Environment" Union of Concerned Scientists, 26 Church Street Cambridge MA 022238, 1991.

[3] Li, H., Andrews, G.E., Daham, B., Bell, M.C., Tate, J.E., Ropkins, K., "Impact of Traffic Conditions and Road Geometry on Real World Urban Emissions using a SI Car", SAE Technical Papers, Document Number: 2007-01-0308, 2007.

[4] Li, H., Andrews, G.E., Khan, A. A., Savvidis, D., Daham, B., Bell, M., Tate, J., Ropkins, K., "Analysis of Driving Parameters and Emissions for Real-World Urban Driving Cycles Using an On-Board Measurement Method for a EURO 2 SI Car”, SAE Technical Papers, Document Number: 2007-01-2066, 2007.

[5] Mierlo, J.V, G. Maggetto, E. Burgwal, and R. Gense. Driving Style and Traffic Measures-Influence on Vehicle Emissions and Fuel Consumption. J of Automobile Engineering, v.218 part D:46-50, 2004.

[6] Treatise UK. Treatise Ecodriving manual: Smart, efficient driving techniques, 2007. http://www.treatise.eu.com/downloads-uk.html

[7] EcoDriven. European Campaign On improving DRIVing behaviour, ENergy-efficiency and traffic behaviour (ECODRIVEN) - Benefits of EcoDriving, 2006. http://www.ecodrive.org/Benefits-of-ecodriving.277. 0.html

[8] United Nations Framework Convention on Climate Change. The First Ten Years. ISBN 92-9219-010-5, 2004.

[9] Kyoto Protocol to the United Nations Framework Convention on Climate Change, 1998.

[10] Green Paper: Towards a new culture for urban mobility, 2007.

[11] European Federation for Transport and Environment, 2007. http://transportenvironment.org

[12] Frey, H.C., A. Unal, and J. Chen, Recommended Strategy for On-Board Emission Data Analysis and Collection for the New Generation Model, Prepared by North Carolina State University for the Office of Transportation and Air Quality, U.S. Environmental Protection Agency, Ann Arbor, MI. February 2002.

[13] Vermuelen, R. J., TNO Report 2006: The effects of a range of measures to reduce the tail pipe emissions and/ort he fuel consumption of modern passenger cars on petrol and diesel. http://www.ecodrive.org/ Downloads.203.0.html

[14] Wilbers, P., Eco-Driving in Netherlands: The smart driving style. Energy Forum, Pamplona, Spain, 2005.

[15] WS31 / WS42 Position Sensors http://www.asm-sensor.com/asm /homepage.php

[16] MTi-Miniature Attitude and Heading sensor. http://www.xsens.com /en/products/machine_motion/mti.php

[17] DAQP-BRIDGE-B Module (revision 2). Technical reference manual. http://www.dewetron.com/products/ 
[18] USB Bus-Powered M Series Multifunction DAQ. National Instruments. http://sine.ni.com/

[19] LabVIEW Development Systems (2008). http://www.ni.com/labview

[20] MySQL 5.1 Reference Manual. MySQL AB, 2008 Sun Microsystems, Inc. http://www.mysql.com 